Varietät des Tolubalsambaumes, und Liquidambar styraciflua nur eine solche von $\mathrm{L}$. orientalis.

Gewisse Differenzen in den Harzprodukten mögen übrigens auch auf die verschiedene Art der Gewinnung zurückzuführen sein, denn es ist natïrlich nicht gleichgültig ob ein Baum stark oder schwach verwundet, ob er nur durch Einschnitte verletzt (Tolubalsambaum), oder geklopft und geschwelt wird (Perubalsambaum). Ob chemische Unterschiede $\mathbf{z w i s c h e n}$ den nach verschiedener Methode von der gleichen Art gewonnenen Harzprodukten wirklich bestehen, werde ich durch Versuche zu ermitteln suchen.

\title{
Ueber die Jodsäure als jodoxydimetrisches Reagens.
}

\author{
Von E. Rupp.
}

(Eingegangen den 22. II. 1905.)

Die Jodsäure ist nach den Untersnchungen von Ditte ${ }^{1}$ ) ein energisches Oxydationsmittel, welches im Sinne der Gleichung

$$
2 \mathrm{HJO}_{\mathrm{B}}=2 \mathrm{~J}+\mathrm{H}_{2} \mathrm{O}+\mathrm{O}_{\mathrm{B}}
$$

unter Abspaltung von freiem Jod fast seinen gesamten Sauerstoffinhalt $\mathrm{zu}$ verausgaben vermag.

Es sollte darum dieses Präparat, welches titrimetrisch außerordentlich scharf bestimmbar ist,

$$
\begin{gathered}
\mathrm{HJO}_{3}+5 \mathrm{HJ}=6 \mathrm{~J}+3 \mathrm{H}_{2} \mathrm{O}, \\
2 \mathrm{~J}+2 \mathrm{Na}_{2} \mathrm{~S}_{2} \mathrm{O}_{8}=2 \mathrm{NaJ}+\mathrm{Na}_{2} \mathrm{~S}_{4} \mathrm{O}_{6},
\end{gathered}
$$

als jodoxydimetrisches Agens hb̈chst brauchbar erscheinen, umsomehr als die oxydative Wirkung durch die Anwendbarkeit erhöhter Temperaturen einer erheblichen Steigerung fähig sein mußte, wäbrend die indirekte Oxydimetrie mit Jod nur äußerst eng begrenzte Temperatursteigerungen zuläßt.

Die Jodsäure wurde in diesem Sinne erstmals von Schwicker ${ }^{2}$ ) verwendet, der die Erfahrung machte, daß selbige "fast nur zur Bestimmung der schwefligen Säure zu gebrauchen ist ${ }^{u}$.

Dieses Resultat ist ein sehr überraschendes. Ich versuchte daher in Gemeinschaft mit Herrn Hartmann die Gründe zu ermitteln, welche die Verwertbarkeit dieses Oxydationsmittels für die Titrimetrie so außerordentlich beschränken. Da die oxydimetrischen Bestimmungen

1) Liebig's Ann. 156, 336.

2) Chem.Ztg. 15, 845 . 
in der Weise erfolgen sollten, daß das durch das Untersuchungsmaterial ans einem Ueberschusse von Jodsäure freigemachte Jod verjagt und der Ueberschuß von Jodsäure gemessen wurde, so wurde zanächst festgestellt, daß Jodsäure sich bei Siedehitze quantitativ erhält:

1. in schwefelsaurer Lösung,

2. in Schwefelsäure und freies Jod enthaltender Lösung, und daß

3. Jodat in neutraler Lösung bei Gegenwart von Jodid titerbeständig bleibt.

$1 \mathrm{ccm}$ Kaliumjodatlösung vom Titer $10,55 \mathrm{ccm}$ n/10 Thiosulfat erforderte mit Wasser verdünnt:

\begin{tabular}{|c|c|c|c|}
\hline & $\begin{array}{c}\text { nach einer } \\
\text { Erhitzungsdauer } \\
\text { von }\end{array}$ & unter Zusatz von & n/10 Thiosulfat \\
\hline $\begin{array}{c}\text { Auf dem } \\
\text { Wasserbade }\end{array}$ & $\begin{array}{lc}15 & \text { Minuten } \\
30 & n \\
60 & n \\
30 & n \\
20 & n \\
40 & n\end{array}$ & 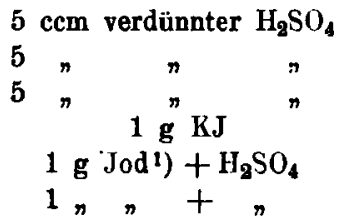 & $\begin{array}{l}10,53 \mathrm{ccm} \\
10,55 " \\
10,54 " \\
10,55 " \\
10,58 \% \\
10,55 ?\end{array}$ \\
\hline $\begin{array}{c}\text { Auf freier } \\
\text { Flamme }\end{array}$ & 30 & $1 n n+n$ & $10,59 \quad n$ \\
\hline
\end{tabular}

Die erforderlichen Vorbedingungen für die Durchführbarkeit von Resttitrationen waren somit gegeben.

Es wurden nunmehr Titrationsversuche mit arseniger Säure und Rhodanwasserstoffsäure ausgeführt. Diese lassen erkennen, daß die Anwendungsmöglichkeit der Jodsäure für oxydimetrische Zwecke dadurch so außerordentlich beschränkt wird, daß die aus dem zu analysierenden Objekte hervorgehenden Oxydations-bezw. Zerfallprodukte indifferent sein müssen gegen freies Jod, gegen Jodwasserstoff und Jodkalium.

\section{Arsenige Säure.}

Zu nachstehenden Versuchsreihen diente eine Kaliumjodatlösung vom Jodwerte 10,55 pro $1 \mathrm{ccm}$ und eine Arsenigsäurelösung mit einem Gehalte von $0,028028 \mathrm{~g} \mathrm{As}_{2} \mathrm{O}_{3}$ in $1 \mathrm{ccm}$. Die $\mathrm{As}_{2} \mathrm{O}_{3}$-Lösung wurde in die mit verdünnter Schwefelsäure angesäuerte Jodatlösung gegossen und nach der momentan erfolgten Umsetzung das Reaktionsgemisch auf ein bestimmtes Volum verdunnt. Aliquote Teile hiervon wurden

1) Das Jod war behufs Befreiung von HJ zuvor solange mit verdünnter $\mathrm{H}_{2} \mathrm{SO}_{4}$ gewaschen worden bis Jodstärke keine Bläuung mehr hervorbrachte. 
zur Verjagung von ausgeschiedenem bezw. in die Lösung gegangenem freien Jod auf dem Wasserbade erhitzt und hierauf der Jodsăureüberschuß bestimmt. Aus diesem berechnet sich der Thiosulfatverbrauch fur $\mathrm{As}_{2} \mathrm{O}_{3}$ wie folgt:

$$
\begin{aligned}
& \mathrm{As}_{8} \mathrm{O}_{3}+2 \mathrm{O}=\mathrm{As}_{9} \mathrm{O}_{6}, \\
& 2 \mathrm{HJO}_{8}=\mathrm{H}_{2} \mathrm{O}+\mathrm{J}_{2}+5 \mathrm{O} \text {, } \\
& \frac{\mathrm{HJO}_{8}+\left(\mathrm{HJ}^{5}=\mathrm{J}_{6}+3 \mathrm{H}_{2} \mathrm{O},\right.}{5 \mathrm{As}_{2} \mathrm{O}_{3}=4 \mathrm{HJO}_{3}=24 \mathrm{~J}}
\end{aligned}
$$

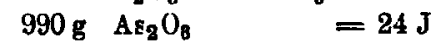

$$
\begin{aligned}
& 40,83 \mathrm{~g} "=127 \mathrm{~g} \mathrm{~J} \\
& 0,004083 \mathrm{~g} n=1 \mathrm{~cm} / 10 \text { Thiosulfat. }
\end{aligned}
$$

Angewandt wurden $5 \mathrm{ccm} \mathrm{As}_{2} \mathrm{O}_{8}, 10 \mathrm{ccm} \mathrm{KJO}_{8}$, aut das Volum von $100 \mathrm{ccm}$ gebracht und hiervon $10 \mathrm{ccm}$ titriert, welche an $\mathrm{n} / 10$ Thiosulfat $7,2-7,4 \mathrm{ccm}$ erforderten. Theoretisch sollten gebraucht werden $7,12 \mathrm{ccm}$, da $0,14014 \mathrm{~g} \mathrm{As}_{2} \mathrm{O}_{3}=34,3 \mathrm{ccm}$ n/10 Thiosulfat entsprechen. Es warden also zu hohe Jodsäurewerte wiedergefunden, die nur darauf zurückgeführt werden können, $d a B$ während der zur Verflüchtigung freien Jods dienenden Erhitzung wiederum in umgekehrtem Prozesse ans Arsensäure + Jod hervorging Jodsäure + Arsenige Säure.

Beweisend hierfür ist folgende Versuchsreihe bei der die Reaktionsgemische aus $5 \mathrm{ccm} \mathrm{KJO}_{3}$, 5 ccm $\mathrm{As}_{2} \mathrm{O}_{3}+\mathrm{H}_{2} \mathrm{SO}_{4}+\mathrm{H}_{2} \mathrm{O}$ samt allem ausgeschiedenen Jod verschieden langer und hoher Erhitzung ansgesetzt wurden. Der berechnete $n / 10$ Thiosulfatverbranch für über-

\begin{tabular}{|c|c|c|c|c|c|c|c|c|c|}
\hline 6 & $n$ & & $\begin{array}{l}1 / 2 \\
5 \mathrm{M}\end{array}$ & inuten & langem & Erhitzen & & & Fener \\
\hline & & $n$ & 10 & $"$ & $n$ & 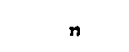 & $n$ & \# & $n$ \\
\hline & $n$ & $n$ & & ündige & Em Erhit & tzen auf $f$ & & Feuer & $\pi$ \\
\hline
\end{tabular}
schüssige Jodsäure beläuft sich hierbei auf $20,55 \mathrm{ccm}$. Gebraucht wurden:

23,2 ccm nach 1/4 stündigen Erhitzen auf dem Wasserbade

unter jeweiliger Ergănzung des verdampften Wassers. Um jegliche den rückläufigen Prozeß außerordentlich begünstigende Erhitzung zu umgehen, wurde nunmehr versucht, durch Titration des freigemachten Jods nach erfolgter Abstumpfung der Schwefelsăure mit Natrium. bikarbonat und durch Titration des freien Jods + überschilssiger Jodsäure nach erfolgtem $\mathrm{KJ}-\mathrm{Znsatz}$ die $\mathrm{As}_{2} \mathrm{O}_{3}$. Menge zu bestimmen.

$$
\begin{aligned}
\text { I. } 2 \mathrm{HJO}_{8} & =2 \mathrm{~J}+\mathrm{H}_{2} \mathrm{O}+\mathrm{O}_{5} \\
5 \mathrm{As}_{2} \mathrm{O}_{3} & =4 \mathrm{HJO}_{8} \\
\hline 990 \mathrm{~g} \mathrm{As}_{2} \mathrm{O}_{8} & =4 \mathrm{~J} \\
247,5 \mathrm{~g} n & =127 \mathrm{~g} \mathrm{~J} \\
0,02475 \mathrm{~g} & =1 \mathrm{ccm} \mathrm{m} / 10 \text { Thiosulfat. }
\end{aligned}
$$




$$
\text { II. } \begin{aligned}
5 \mathrm{As}_{2} \mathrm{O}_{8} & =4 \mathrm{HJO}_{3}=24 \mathrm{~J}+4 \mathrm{~J} \\
990 \mathrm{~g} \mathrm{As}_{2} \mathrm{O}_{\mathrm{B}} & =28 \mathrm{~J} \\
35,35 \mathrm{~g} \quad n & =127 \mathrm{~g} \mathrm{~J} \\
0,003535 \mathrm{~g} n & =1 \mathrm{ccm} \mathrm{n} / 10 \text { Thiosulfat. }
\end{aligned}
$$

Genügend konstante Werte konnten auch hierbei nicht erzielt werden, so daß endlich noch die Ausschuttelung treien Jods mit Chloroform probiert wurde. Wohl wurden auf diesem Wege bei reichlich bemessenen Mengen von wassergesattigtem Chloroform und einer wiederholten Anssch attelung befriedigende Befunde erzielt, allein die Hartnäckigkeit mit der die letzten Spuren von Jod festgehalten werden, lassen auch diesen Umweg nicht als zuverlăssig und einfach erscheinen. Es beanspruchten $10 \mathrm{ccm}$ des wie in der ersten Versuchsreihe zusammengestellten Reaktionsgemisches an $\mathrm{m} / 10$ Thiosulfat

$7,25 \mathrm{ccm}$ nach Ausschüttelung mit mäBig viel Chloroform $7,18-7,20 \eta n$ n sehr viel Chloroform $7,10-7,15, n$ dreimaliger Ausschüttelung mit Chloroform Theoretischer Thiosulfatrerbrauch $7,12 \mathrm{ccm}$.

\section{Rhodanwasserstofi.}

Ein weniger rom Chemiker als vom Physiologen getibter Nachweis von Rhodanwasserstoff bernht auf der Ausscheidung von Jod aus Jodsăure bezw. schwefelsaurer Jodatlősung.

Nach der Gleichang

$$
6 \mathrm{HCNS}+6 \mathrm{HJO}_{8}+2 \mathrm{H}_{2} \mathrm{O}=5 \mathrm{H}_{8} \mathrm{SO}_{4}+5 \mathrm{HCN}+6 \mathrm{~J}
$$

entsprechen

$$
\begin{aligned}
5 \mathrm{KCNS} & =6 \mathrm{HJO}_{8} \\
\mathrm{HJO}_{8}+5 \mathrm{HJ} & =6 \mathrm{~J} \\
\hline 5 \mathrm{KCNS} & =36 \mathrm{~J} \\
485 \mathrm{~g} \quad & =36 \mathrm{~J} \\
13,47 \mathrm{~g} " & =127 \mathrm{~g} \mathrm{~J} \\
0,001347 \mathrm{~g} \rrbracket & =1 \mathrm{~cm} / 10 \text { Thiosulfat. }
\end{aligned}
$$

Es warden $4 \mathrm{ccm}$ einer Rhodankaliumlösung, welche 0,02387 g KCNS in $1 \mathrm{ccm}$ enthielt und $10 \mathrm{ccm}$ Jodatlösung ( $=105,5 \mathrm{ccm} n / 10$ Thiosulfat) gemischt, sodann verdunnte Schwefelsäure hinzngefügt, worauf momentane Jodabscheidung erfolgt. Das Volum warde nun auf $100 \mathrm{ccm}$ ergănzt und in $10 \mathrm{ccm}$ hiervon nach der Verjagung freien Jods anf dem Wasserbade in bekannter Weise der JodatuberschuB bestimmt. Dabei warden an $n / 10$ Thiosulfat beansprucht:

Nach 5 Minuten langem Erhitzen 3,45-3,60 ccm

\begin{tabular}{cccccc|c}
$n$ & 10 & $n$ & $n$ & $n$ & $3,35-3,40$ & $n$ \\
$n$ & 30 & $n$ & $n$ & $n$ & $2,80-3,20$ & $n$ \\
$n$ & 60 & $n$ & $n$ & $n$ & $2,70-2,90$ & $n$
\end{tabular}


Diese Inkonstanz deutet wiederam auf Nebenreairtionen hin, hier jedoch im umgekehrten Sinne der bei $\mathrm{As}_{2} \mathrm{O}_{3}$ gemachten Beobachtungen. Es wurde $\mathrm{HJO}_{B}$ in ansteigender Menge verbraucht. Diese Komplikation wird hervorgerufen darch nachträgliche Bildang von Jodcyan. Während zunächst das Reaktionsgemisch bei reichlicher Jodabscheidnng einen reinen Blausluregeruch entwickelt, geht beim Stehenlassen und beim Erwärmen mehr und mehr Jod in Lösung. Hierbei tritt der eigen. tümlich scharfe Geruch des Jodcyans auf. Dieser erhält sich mit großer Hartnäckigkeit auch dann noch, wenn die erhitzte Lősung schon längst absolut farblos, also jodfrei geworden. Der Vorgang ist folgender: $\mathrm{HCN}+2 \mathrm{~J}=\mathrm{JCN}+\mathrm{HJ}$.

Dieser sekundär erzeugte Jodwasserstoff wirkt naturgemäß zerstorrend auf Jodsăure ein und daher die beobachteten Unterwerte. Die Versuche, freies Jod möglichst rasch und ehe die JCN-Bildung einsetzt, mit Chloroform auszuschtitteln, mußten als unsicher verworfen werden.

Wenn die vorerwihnten Versuche mit negativem Erfolge verliefen, aus Gründen sekundärer Reaktionen, dann mußte eine Ausschaltung dieser Faktoren positive Resultate ergeben, so z. B. die 'Titration der Ameisensäure, welche völlig indifferente Oxydationsprodukte ergibt

$$
\mathrm{HCOOH}+\mathrm{O}=\mathrm{CO}_{2}+\mathrm{H}_{2} \mathrm{O} \text {. }
$$

In der Tat war dem auch so. Da Ameisensäure durch Jod in bikarbonatalkalischer Lösung nicht oxydierbar ist, so erlæhutert dieser Fall gleichzeitig die Ueberlegenheit der Jodsănre gegenüber dem Jod in Bezug auf deren oxydative Wirkung.

Nach der Umsetzungsformel

$$
\begin{array}{ll}
2 \mathrm{HJO}_{3}+5 \mathrm{HCOOH} & =2 \mathrm{~J}+6 \mathrm{H}_{2} \mathrm{O}+5 \mathrm{CO}_{8} \\
\mathrm{HJO}_{8}+5 \mathrm{HJ} & =6 . \mathrm{J} \\
2 \mathrm{HJO}_{8} & =5 \mathrm{HCOOH} \\
2 \mathrm{HJO}_{8} & =12 \mathrm{~J} \\
\hline \mathbf{5} \mathrm{HCOOH} & =12 \mathrm{~J}
\end{array}
$$

sind

$$
\begin{aligned}
230 \mathrm{~g} \mathrm{HCOOH} & =12 \mathrm{~J} \\
19,17_{n}{ }_{n} & =127 \mathrm{~g} \mathrm{~J} \\
0,001917 \mathrm{~g}_{n} & =1 \mathrm{~cm} \mathrm{n/10 \textrm {J }} .
\end{aligned}
$$

In ein verdünntes Gemisch von $10 \mathrm{ccm}$ Jodatlösung (Jodwert $105,6)$ and $5 \mathrm{ccm}$ verdünnter $\mathrm{H}_{2} \mathrm{SO}_{4}$ wurden $2 \mathrm{ccm} \mathrm{N}$.-Ameisensäure gegeben. Zunăchst trat keine Reaktion ein, wohl aber, als das Ganze in wohlverschlossenem Stopselglase ca. 30 Minuten anf dem Wasserbade unter zeitweiliger I tuttung des Stöpsels erhitzt wurde.

$20 \mathrm{ccm}$ der erkalteten Flüssigkeit wurden abgehoben, gelöstes freies Jod durch ca. 10 Minuten lange Erhitzung in einer Schale 
verjagt und der Jodatüberschuß in bekannter Weise gemessen. Hierzu warden an $1 / 10$ Thiosulfat ben 8 tigt $11,5-11,54 \mathrm{ccm}$, im Mittel $11,52 \mathrm{ccm}=57,6$ pro toto $=48,0 \mathrm{ccm}$ pro Ameisenshure $=0,092016 \mathrm{~g}$ HCOOH $=100,1 \%$ der angewandten Menge.

Vergleichsweise führten wir die Bestimmung der Ameisensăure in ganz analoger Weise auch mit Bromsäure durch.

Ueber die Verwendung der Bromsăure als Oxydationsagens in der Maßanalyse liegen Untersuchungen vor von Feit ${ }^{1}$, Feit and Kubierschky ${ }^{2}$ ) und von Schwicker ${ }^{8}$ ). Denselben ist zu entnehmen, daß die Bromsăure bezw. die schwetelsanre Lösung ihrer Salze ein ausgezeichnetes Oxydationsvermögen besitzt und zur quantitativen Bestimmung zahlreicher Stoffe mit Vorteil herangezogen werden kann. Es wurden damit nach dem Ueberschußbestimmungsverfahren analysiert Sulfide, Nitrite, Sulfite, Ferrosalze und Oxalsăure.

Für die Ameisensüure ergaben sich die geeigneten Versuchsbedingungen aus folgendem:

$1 \mathrm{ccm}$ einer ca. $10 \%$ igen Ameisenshurelösung wurde im offenen Erlenmeyer-Kolben mit $10 \mathrm{ccm}$ einer ca. 2,5\% igen Kaliumbromatlosung genau ermittelten Bromatwertes versetzt, mit ca. 5 ccm verdünnter Schwefelsăure angesłuert und auf das Wasserbad verbracht. Mit beginnender Erwarmung tritt Umsetzung ein. Es wird dann mit der Erhitzang solange fortgefahren bis die Flüssigkeit völlig entfärbt and kein Bromgeruch mehr wahrnehmbar ist, was nach ca. einer Stande sicher der Fall ist. Erhitzung in offener Schale wïde rascher zum Ziele fuhren, ist aber zu vermeiden am Verdampfungsverluste von Ameisensäure zu vermeiden. Nach dem Erkalten wird der Bromatuberschuß ebenso zuríckgemessen wie urspringlich der Bromattiter ermittelt wurde, d. h. man verdünnt mit Wasser anf ca. $75-100 \mathrm{ccm}$, setzt einige Kubikzentimeter verdunnte Schwefelsăure and $1-2 \mathrm{~g}$ Jodkalium hinzu, worauf unter Zusatz von Starkeløsung das ansgeschiedene Jod mit $n / 10$ Thiosulfat gemessen wird.

Die Berechnung entspricht den Gleichungen:

$$
\begin{aligned}
2 \mathrm{HBrO}_{8}+5 \mathrm{H} \cdot \mathrm{COOH} & =2 \mathrm{Br}+6 \mathrm{H}_{2} \mathrm{O}+5 \mathrm{CO}_{2} \\
\mathrm{HBrO}_{\mathrm{a}}+6 \mathrm{HJ} & =6 \mathrm{~J}+\mathrm{HBr}+3 \mathrm{H}_{2} \mathrm{O} \\
2 \mathrm{HBrO}_{\mathrm{B}} & =12 \mathrm{~J} \\
2 \mathrm{HBrO}_{\mathrm{g}} & =5 \mathrm{H} \cdot \mathrm{COOH} \\
\text { folglich } 5 \mathrm{H} \cdot \mathrm{COOH} & =12 \mathrm{~J} \\
0,001917 \mathrm{~g} \mathrm{H} \cdot \mathrm{COOH} & =1 \mathrm{ccm} \mathrm{n}_{110} \text { Thiosulfat. }
\end{aligned}
$$

1) Chem.-Ztg. 15, 351.

2) Chem. Zentralbl. 1891, I., 759.

8) Chem.-Ztg. 15, 845. 
Die Titrationsergebnisse mit $10 \mathrm{ccm}$ Bromatlösung $=91 \mathrm{ccm} n / 10$ Thio. sulfat waren:

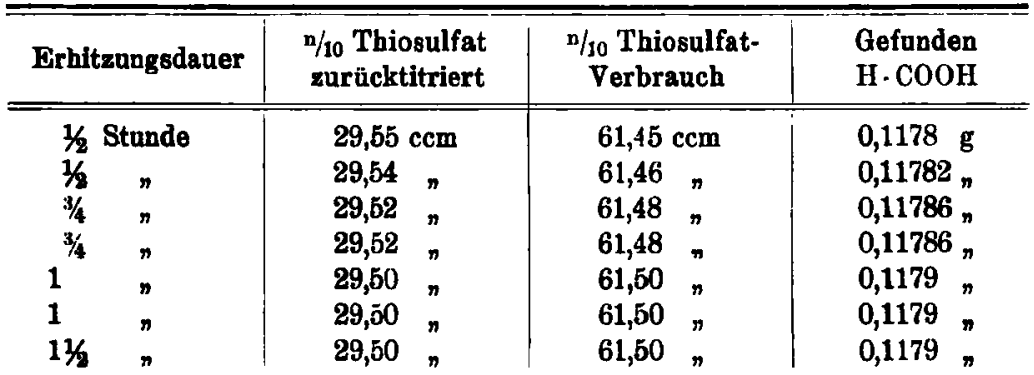

Die acidimetrische Kontrollanalyse ergab einen Normalalkaliverbrauch von $25,63 \mathrm{ccm}$ für $10 \mathrm{ccm}$ Săure $=0,11795 \mathrm{~g} \mathrm{HCOOH}$ in einem Kubikzentimeter Lösung.

Ein Vergleich beider Bestimmungen spricht in jeder Beziehung zu Gunsten der Bromsäure. Da das abgespaltene Brom nicht wieder oxydierbar und leicht flüchtig ist, so fallen die Beschrankungen, welche für die Anwendung von Jodsaure gultig sind, hier vollstandig weg, und wird mandaher die Jodshure als jodometrisches Oxydationsagens überhaupt vollkommen ausschalten.

Noch rascher als Bromsäure tührt in einer Reihe von Fällen Alkalihypobromit zum Ziele. Ameisensäure wird hierdurch, wie bereits berichtet, schon in der Kälte innerhalb 30 Minuten vollkommen oxydiert.

\title{
Deber die titrimetrische Bestimmung von Ammonsalzen mit Alkalihypobromit.
}

\author{
Von E. Rupp und E. Rößler.
}

(Eingegangen den 28. II. 1905.)

Es ist seit langer Zeit bekannt, daß Hypohalogenite auf Ammoniak nnter Entwickelang von Stickstoff einwirken

$$
2 \mathrm{NH}_{8}+3 \mathrm{NaOBr}=3 \mathrm{NaBr}+2 \mathrm{~N}+2 \mathrm{H}_{2} \mathrm{O} \text {. }
$$

Die Frage nach dem quantitativen Verlaufe dieser Reaktion hat eine außerordentlich umfangreiche Literatur gezeitigt, welche die gasvolumetrische Bestimmung von Ammonverbindungen durch Messen des entwickelten Stickstoffvolums im Azotometer zum Gegenstande hat. 